\title{
Hemorrhagic Vestibular Schwannoma: Case Report and Literature Review of Incidence and Risk Factors
}

\author{
Taha Shahbazi ${ }^{1}$, Mohammadmahdi Sabahi ${ }^{2}$, Mahdi Arjipour ${ }^{3}$, Badih Adada ${ }^{4}$, Hamid Borghei-Razavi ${ }^{5}$ \\ 1. Neurosurgery Research Group (NRG), Student Research Committee, Hamadan University of Medical Science, \\ Hamadan, IRN 2. Neurosurgery Research Group (NRG), Student Research Committee, Hamadan University of Medical \\ Sciences, Hamadan, IRN 3. Neurosurgery, Hamadan University of Medical Science, Hamadan, IRN 4. Neurosurgery, \\ Cleveland Clinic Florida, Weston, USA 5. Neurosurgery, Pauline Braathen Neurological Center, Cleveland Clinic \\ Florida, Weston, USA
}

Corresponding author: Mahdi Arjipour, arjipour.nrg@gmail.com

\begin{abstract}
Hemorrhagic vestibular schwannoma (HVS) consisting of acute intratumoral and subarachnoid hemorrhage presents with acute nausea, vomiting, facial numbness, headache, loss of consciousness, and significant functional impairment of the facial and vestibulocochlear nerves. The current case is of a 31-year-old man who was presented with acute left lateral suboccipital headache, vomiting, ataxia, and loss of consciousness. Brain CT revealed a large iso-intense lesion with internal hematoma at the left cerebellopontine angle in association with internal acoustic canal dilation. In addition, MRI confirmed a $32 \times 25 \times 26 \mathrm{~mm}$ vestibular schwannoma (VS) with $20 \times 15 \times 5$ mm intratumoral hematoma. The patient had undergone left lateral suboccipital craniotomy and microscopic tumor resection. Pathological findings revealed that his lesions were VS. The average incidence of HVS is around 2.15 cases per year worldwide. Therefore, HVS incidence in proportion to VS is very low and consequently rare.
\end{abstract}

Categories: Neurology, Neurosurgery, Oncology

Keywords: intratumoral hemorrhage, subarachnoid hemorrhage, vestibular schwannoma, acoustic neuroma, hemorrhage

\section{Introduction}

Vestibular schwannoma (VS), also called acoustic neuroma, is a benign tumor of the vestibulocochlear nerve that has an incidence of 11-13 cases per million every year. It arises from Schwann cells at the ObersteinerRedlich zone [1-3].

Received 08/18/2020 Review began 08/24/2020 Review ended 08/31/2020 Published 09/01/2020

๑) Copyright 2020 Shahbazi et al. This is an open access article distributed under the terms of the Creative Commons Attribution License CC-BY 4.0., which permits unrestricted use, distribution, and reproduction in any medium, provided the original author and source are credited.
Hemorrhagic VS (HVS) is a rare phenomenon. Only limited cases are reported and there has been no definite estimation about HVS incidence until now. When significant HVS occurs, patients may experience acute neurological defects [4]. Considered risk factors include huge tumor size, cystic development, hypointense parts in T2-weighted magnetic resonance (MR) signal, hemosiderin deposition due to intratumoral hemorrhage (ITH), and a history of anticoagulant therapy $[5,6]$.

The treatment is based on surgery, and radiation therapy (RT) and observation are other choices of treatment. There are three standard surgical approaches: retromastoid suboccipital (retrosigmoid), translabyrinthine, and middle fossa approach (MFA). Although surgery is the treatment of choice for VS, there are many different RT techniques including single-session stereotactic radiosurgery (SRS), fractionated conventional RT, fractionated stereotactic RT (FSRT), and proton therapy [7-10].

Herein, we report a case of a patient who was admitted with HVS. Some similar cases have also been reviewed as literature review.

\section{Case Presentation}

All procedures performed in studies involving human participants were in accordance with the ethical standards (code: IR.UMSHA.REC.1399.388) and the 1964 Helsinki Declaration and its later amendments or comparable ethical standards. Of note, informed consent was obtained from the patient in order to use his information for the case presentation. A 31-year-old shepherd man was referred to our center due to loss of consciousness. He was able to communicate and explained his experiences of a sudden severe headache in the left lateral suboccipital (retroauricular) region, as well as left hemi-facial numbness, nausea, and vomiting. After a while, he developed disequilibrium and loss of consciousness instantly after walking a few steps. Neurological examination showed he had facial paresis (House-Brackmann grade II), vertigo, and ataxia, whereas other examinations were normal.

His past medical history revealed he had a history of appendectomy 30 months before current admission. He 


\section{Cureus}

had also developed deep vein thrombosis and pulmonary emboli 18 months ago and had used aspirin after thrombolytic therapy. Moreover, he had a history of surgical removal of lung hydatid cyst 12 months ago as well as a history of headache for three years, which was intractable to any treatment. His prothrombin time (PT), partial thromboplastin time (PTT), and international normalized ratio (INR) were normal, and no abnormal findings were reported in lab results.

Brain CT revealed extra-axial lesion at the left cerebellopontine angle (CPA) region with an intralesion hematoma that expanded tumor volume (Figure 1A). The left internal acoustic canal (IAC) was dilated in comparison with the right side. The brain stem and fourth ventricle were compressed with the mass effect of hemorrhagic tumor. There was some ventriculomegaly but no periventricular edema and hydrocephalus.

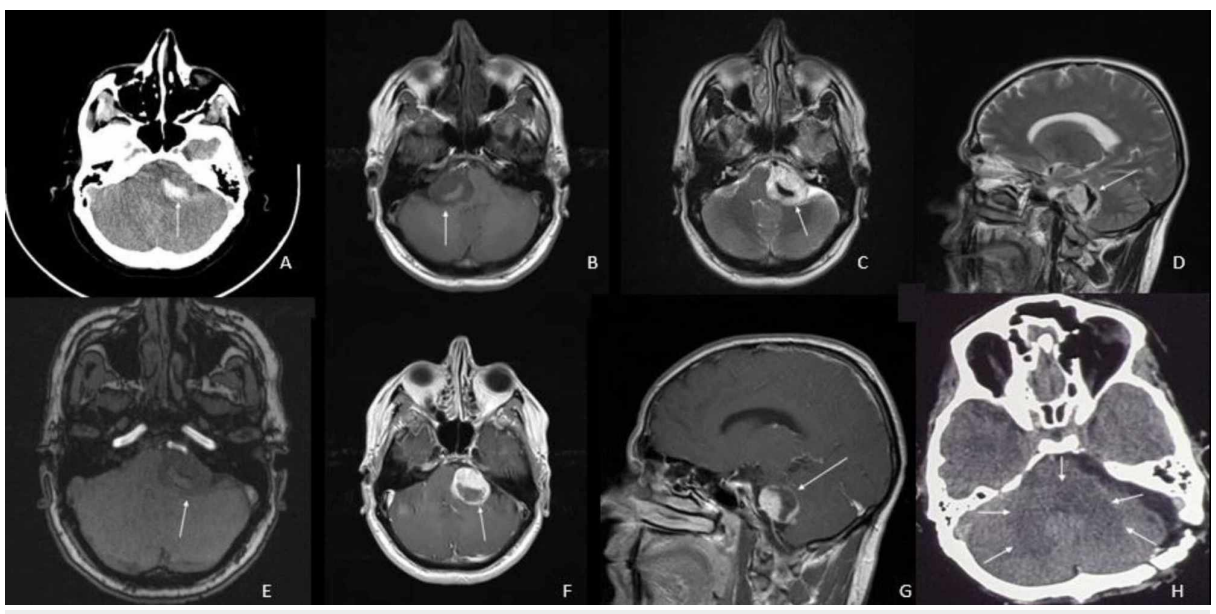

\section{FIGURE 1: Patient's Preoperative Imaging}

(A) Brain CT shows an extra-axial lesion at the left CPA region with intralesion hematoma. (B and C) Axial T1and T2 -weighted images, respectively, show extra-axial lesion, which was hypointense on T1-weighted images and hyperintense on T2-weighted images. Intralesional hematoma is iso- and hypointense on T1- and T2-weighted images, respectively. (D) Sagittal T2-weighted image. (E) Axial MRI source image. (F and G) Contrast T1-weighted axial and sagittal images show homogenous avid tumor enhancement. $(\mathrm{H})$ Contrast CT show postoperation changes and tumor resection with retrosigmoid approach.

In the MRI, $32 \times 25 \times 26 \mathrm{~mm}$ VS at the left CPA with extension to IAC was seen. The lesion was hypo- and hyperintense on T1-weighted image (T1WI) and T2-weighted image (T2WI), respectively (Figures $1 B-1 D$ ) and enhanced dens homogenous on contrast T1WI (Figures 1F, 1G). Intralesion hematoma measuring $20 \mathrm{x}$ $15 \times 5 \mathrm{~mm}$ was iso- and hypointense on T1WI and T2WI, respectively, without T1WI enhancement contrast (Figure 1).

The patient was operated at semi-lateral supine position with left lateral suboccipital craniotomy and microscopic retrosigmoid approach. Intratumoral hematoma was seen during tumor resection. The tumor was totally resected, and the facial nerve was preserved. The patient's postoperation course was uneventful and without any new neurological deficit (Figures $2 A, 2 B$ ).
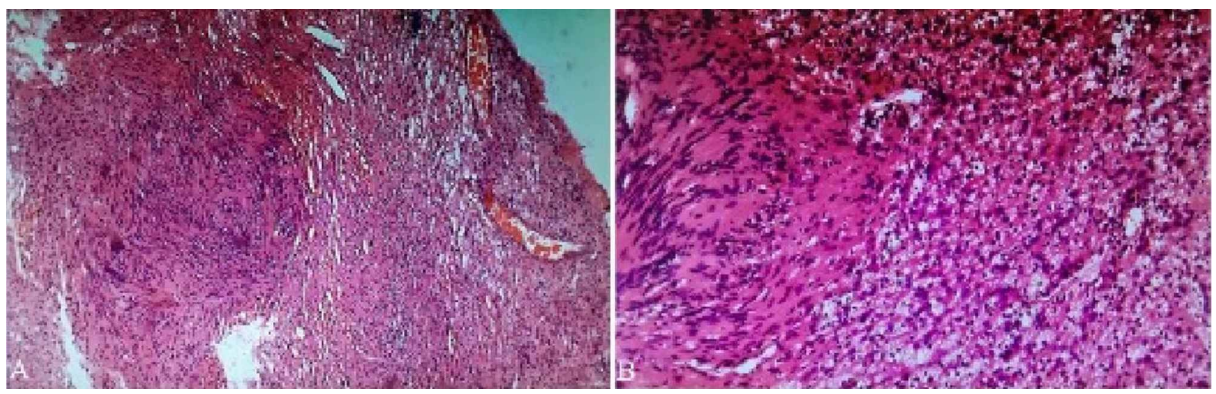

\section{FIGURE 2: Lesion Microscopic View}

(A) Low power field. (B) High power field microscopic view shows vestibular schwannoma. Antoni A and B patterns are seen in the collagenous background. In the hypercellular Antoni A areas, intersecting fascicles consisting of spindle cells with buckled nuclei are seen. These cells form Verocay bodies with nuclear palisading, making alternating bands of nuclear and anuclear areas. In the hypocellular Antoni B areas, the prominent myxoid extracellular matrix takes the spindle cells apart. 
In the hematoxylin and eosin (H \& E) staining, hypercellular (Antoni A) and hypocellular (Antoni B) areas are seen. Dense Antoni A areas consisting of interlacing bundles of spindle cells with oval nuclei, eosinophilic cytoplasm, and indistinct cytoplasmic borders are also seen. Hypocellular dense Antoni B areas are composed of haphazardly arranged spindle cells in loose myxoid collagen fibers. Blood vessels with hyalinized walls were visible.

\section{Discussion}

The VS is an uncommon intracranial tumor and frequently presents with chronic hearing loss, headache, tinnitus, disequilibrium, and facial numbness, whereas acute overt hemorrhage including subarachnoid hemorrhage (SAH) and ITH as its first presentation is quite rare [11].

Of all intracranial hemorrhages, $1-11 \%$ belong to hemorrhagic brain tumors. SAH that arises from brain tumors accounts for $0.4 \%$ of all cases of SAH. Furthermore, 1.7- $10 \%$ of brain tumors cause intracerebral hemorrhage [12]. ITH generally occurs in $11 \%$ of all cranial tumors, and its occurrence in glioblastoma multiforme, pituitary adenomas, choriocarcinomas, oligodendrogliomas, choroid plexus papillomas, and meningiomas is also prevalent.

According to Mathkour et al.'s literature review, there were only 48 cases of VS secondary to ITH, whereas in accordance with our review of the literature (Table 1), there were 97 HVS cases since 1974 including our case [13].

Based on the reported cases, the average incidence of HVS worldwide is approximately only 2.15 cases per year; therefore, HVS is a quite rare entity among VS tumors.

Some studies have reported hemorrhagic cystic VS (CVS) and therefore it can be considered as a risk factor for HVS (Table 1). Although our case's tumor was not of cystic type, CVS has been characterized with faster expansion rate than the solid ones, rapid nerve involvement, and development of variable symptoms. CVS is more commonly demonstrated with fluid-fluid levels and hemosiderin deposition on imaging. Moreover, it has been argued that ITH can result in the formation of the cyst [14].

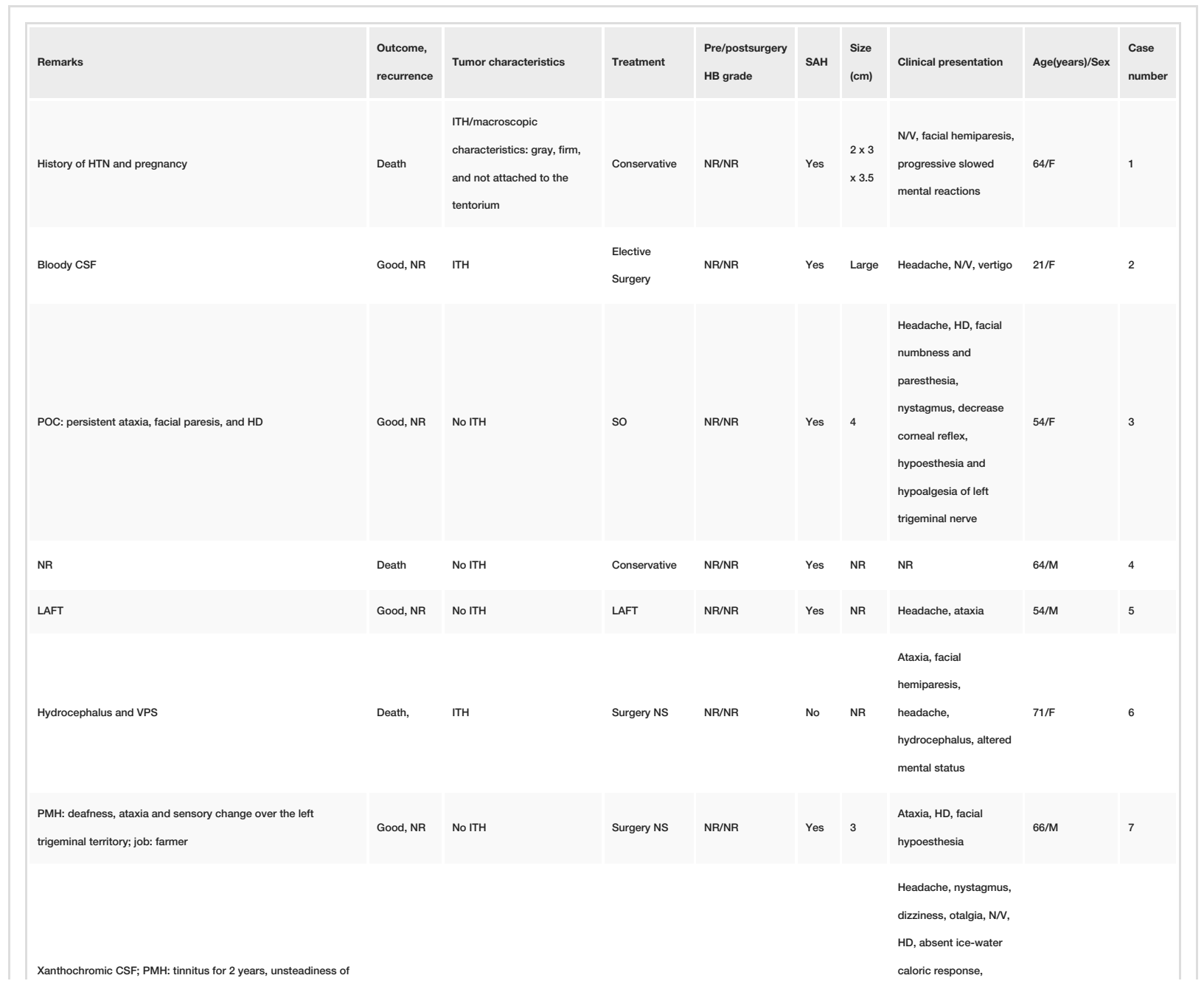




\section{Cureus}

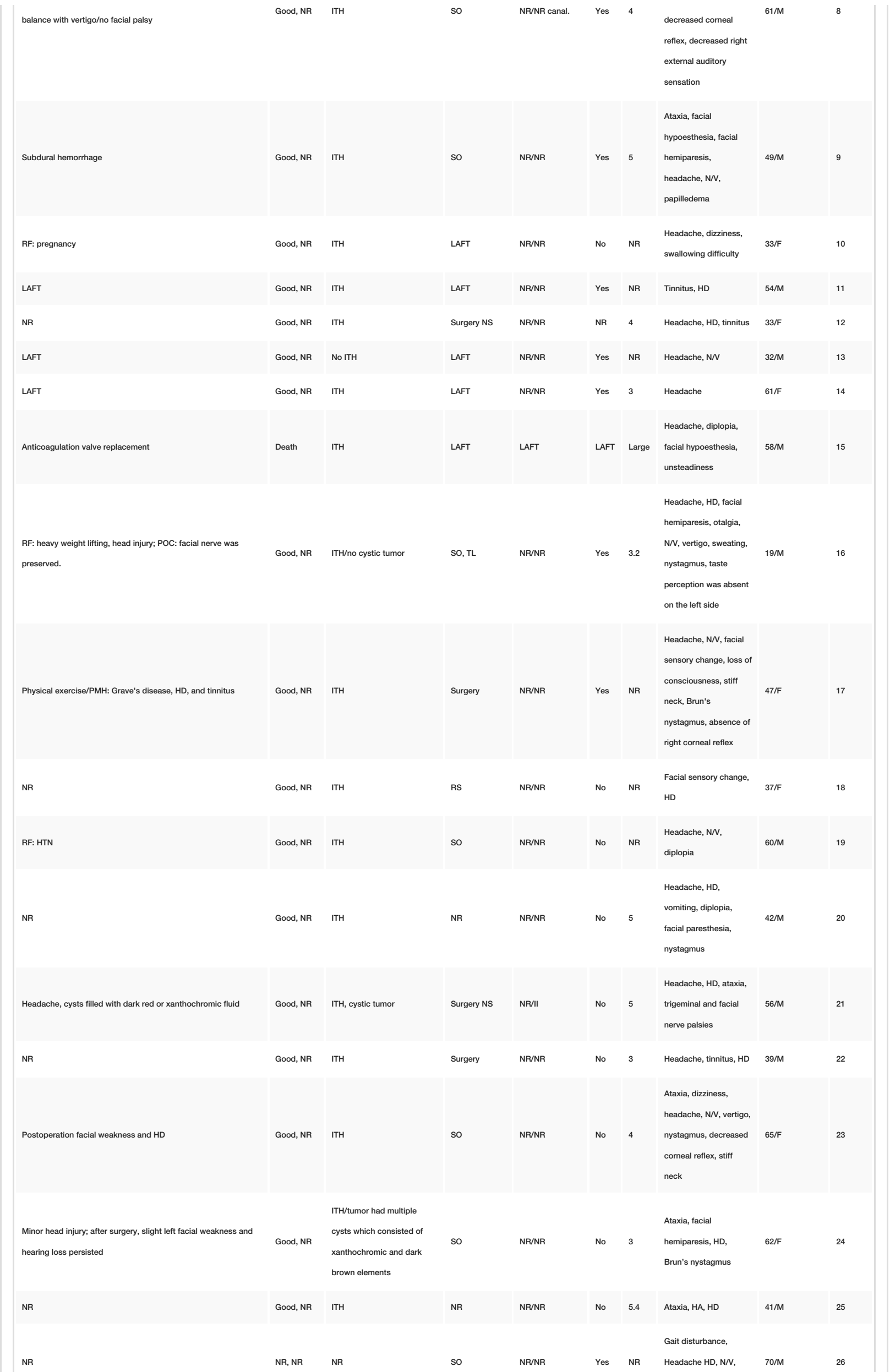




\section{Cureus}

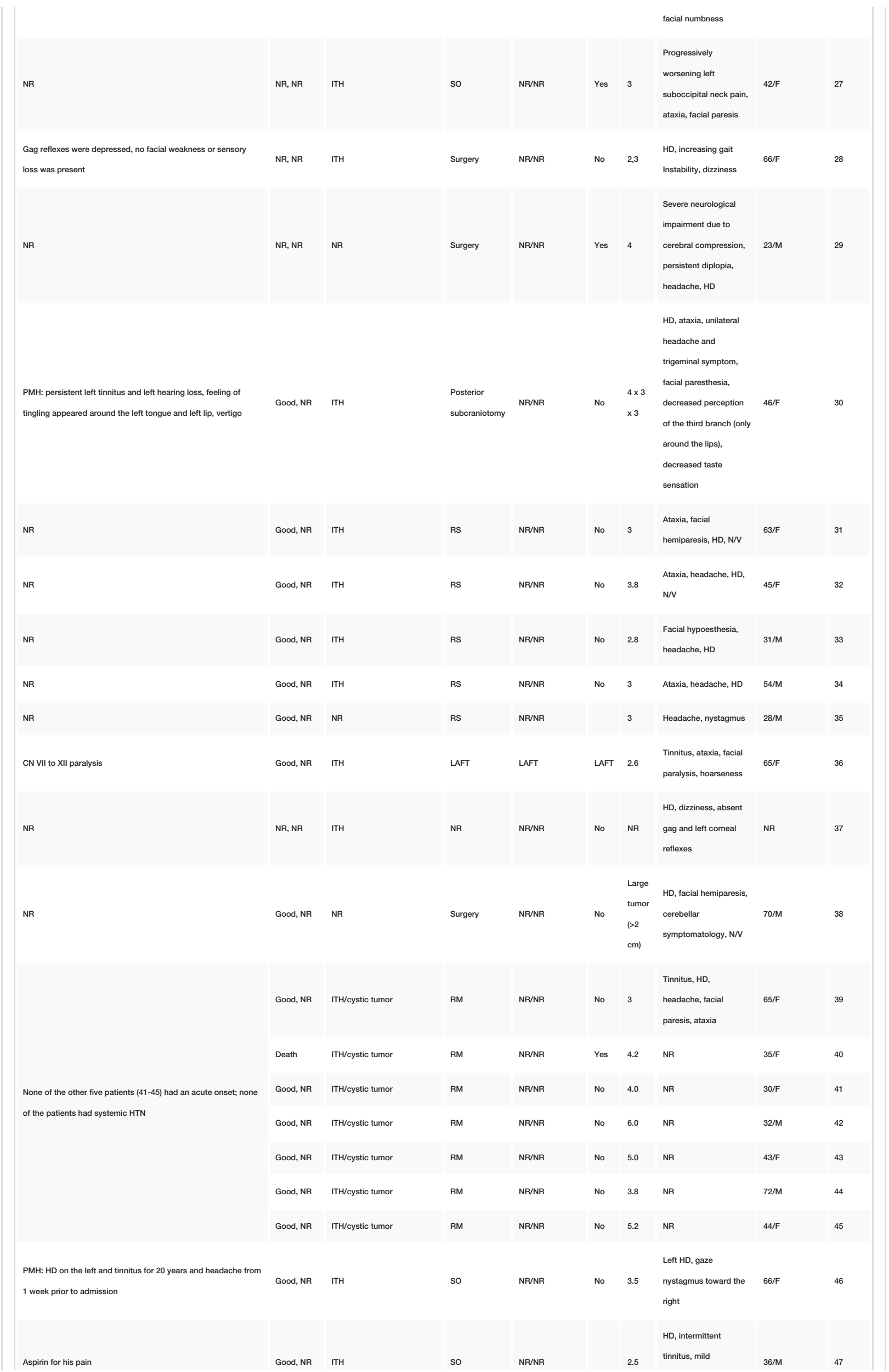




\section{Cureus}

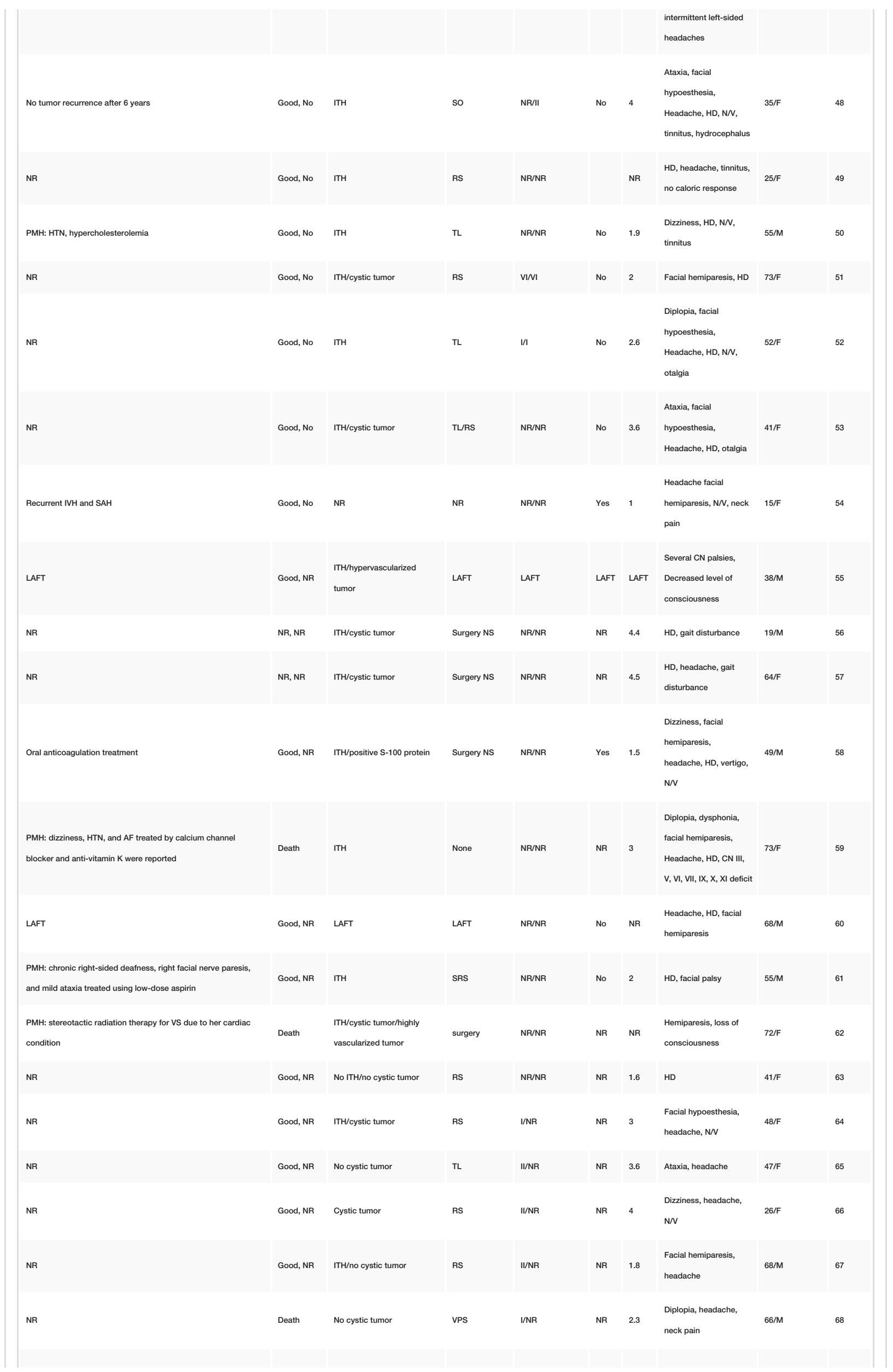




\section{Cureus}

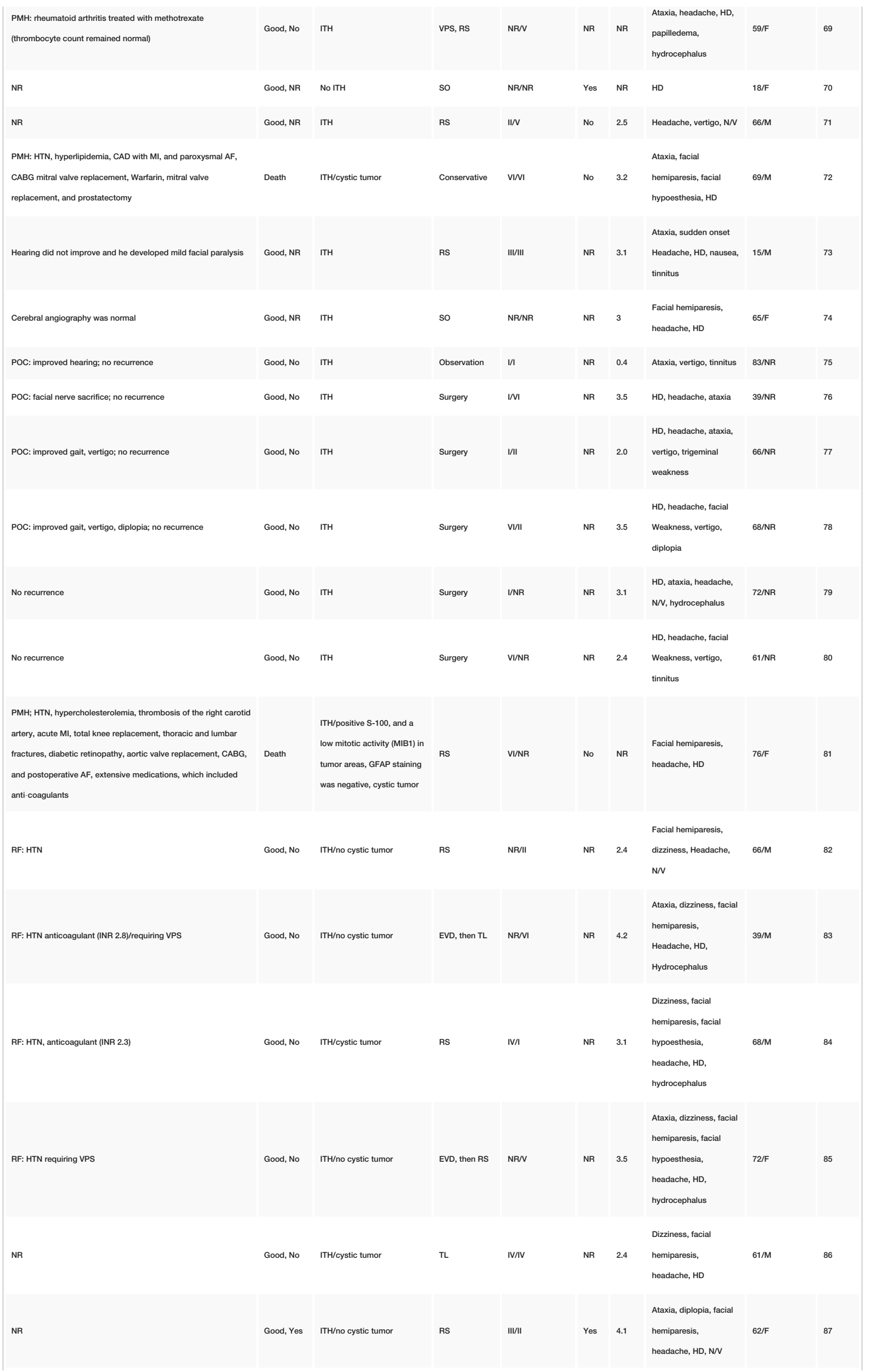




\section{Cureus}

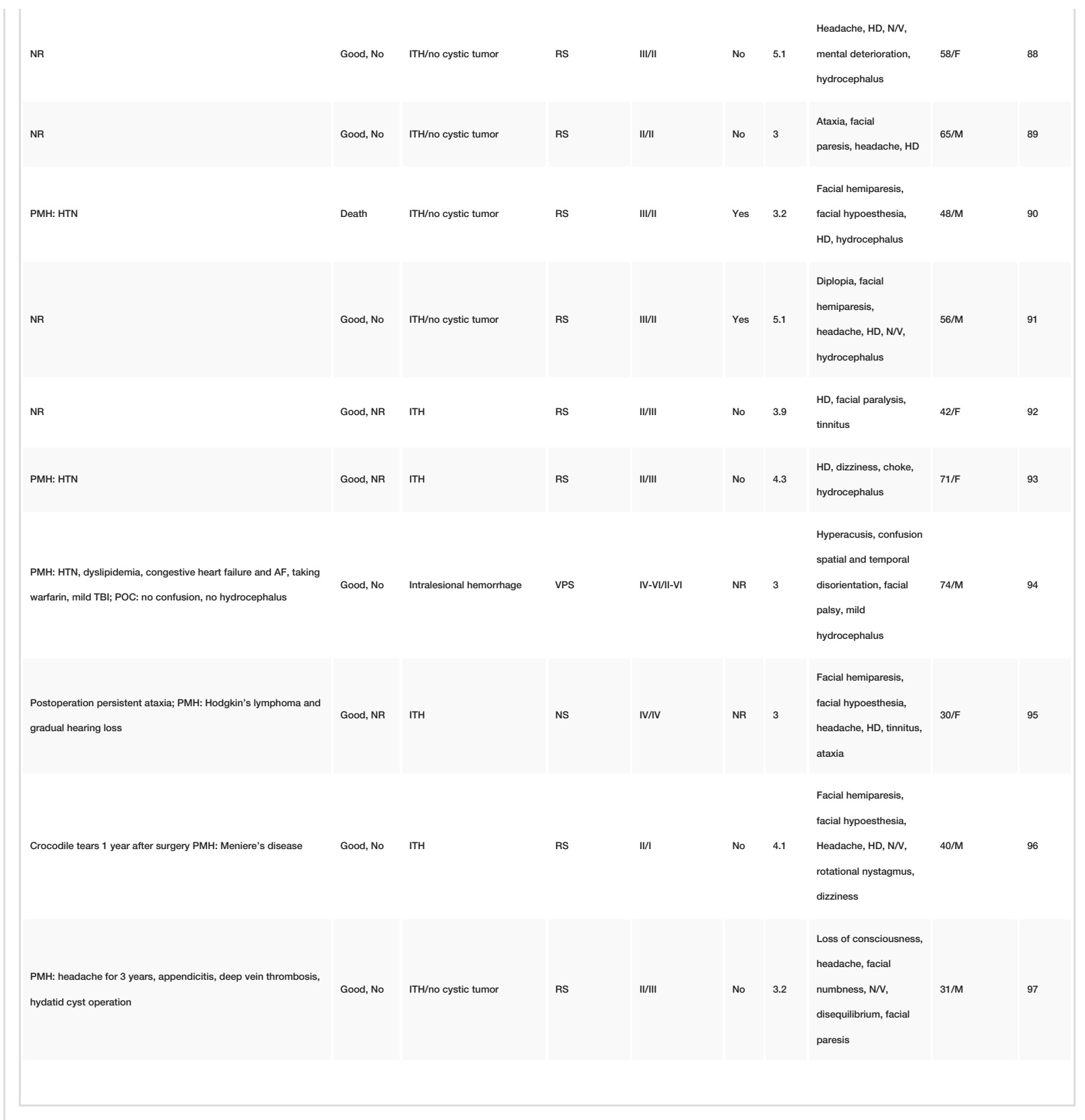

\section{TABLE 1: Reported Hemorrhagic Vestibular Schwannoma}

Abbreviations: AF, arterial fibrillation; $C A B G$, coronary artery bypass grafting; CAD, coronary artery disease; CN, cranial nerve; CSF, cerebrospinal fluid; EVD, external ventricular drain; F, female; GFAP, glial fibrillary acidic protein; HB, House-Brackmann grade; HD, hearing disturbance; HTN, hypertension; ITH, intratumoral hemorrhage; IVH, intraventricular hemorrhage; LAFT, lack of access to full text; M, male; MI, myocardial infarction; NR, not reported; NS, not otherwise specified; N/N, nausea/vomiting; PMH, past medical history; POC, postoperative conditions; RF, risk factor; RM, retromastoid; RS, retrosigmoid; SAH, subarachnoid hemorrhage; SO, suboccipital craniotomy; SRS, stereotactic radiosurgery; TBI, traumatic brain injury; TL, translabyrinthine; VPS, ventriculoperitoneal shunt; VS, vestibular schwannoma

It is assumed that anticoagulant therapy could be a potential risk factor for HVS. In accordance with other reports, our case had a history of anticoagulant (aspirin) therapy $[15,16]$.

Our patient had high physical activities and he was a shepherd. The patients reported by others had a history of pregnancy, farming, heavy weight lifting, strenuous exercise, and hypertension [17-20]. As a result, these items can also be considered as potential risk factors for HVS.

\section{Conclusions}

Micro-ITH may happen more commonly in VS, but clinically significant hemorrhage has a very rare occurrence. HVS risk factors consist of huge tumor size, cystic development, history of anticoagulant therapy, and strenuous activities. 


\section{Additional Information \\ Disclosures}

Human subjects: Consent was obtained by all participants in this study. Hamadan University of Medical Science issued approval IR.UMSHA.REC.1399.388. All procedures performed in studies involving human participants were in accordance with the ethical standards (code: IR.UMSHA.REC.1398.982) and with the 1964 Helsinki declaration and its later amendments or comparable ethical standards. Conflicts of interest: In compliance with the ICMJE uniform disclosure form, all authors declare the following: Payment/services info: All authors have declared that no financial support was received from any organization for the submitted work. Financial relationships: All authors have declared that they have no financial relationships at present or within the previous three years with any organizations that might have an interest in the submitted work. Other relationships: All authors have declared that there are no other relationships or activities that could appear to have influenced the submitted work.

\section{References}

1. Fisher JL, Pettersson D, Palmisano S, et al.: Loud noise exposure and acoustic neuroma . Am J Epidemiol. 2014, 180:58-67. 10.1093/aje/kwu081

2. Xenellis JE, Linthicum FH, Jr: On the myth of the glial/schwann junction (Obersteiner-Redlich zone): origin of vestibular nerve schwannomas. Otol Neurotol. 2003, 24:1. 10.1097/00129492-200301000-00001

3. Carlson ML, Link MJ, Wanna GB, Driscoll CL: Management of sporadic vestibular schwannoma. Otolaryngol Clin North Am. 2015, 48:407-22. 10.1016/j.otc.2015.02.003

4. Niknafs YS, Wang AC, Than KD, et al.: Hemorrhagic vestibular schwannoma: review of the literature . World Neurosurg. 2014:751-6. 10.1016/j.wneu.2013.02.069

5. Ganslandt O, Fahrig A, Strauss C: Hemorrhage into cystic vestibular schwannoma following stereotactic radiation therapy. Zentralbl Neurochir. 2008, 69:204-6. 10.1055/s-2008-1077074

6. Ishii K, Takahashi S, Matsumoto K, et al.: Hemorrhage and abnormal veins in acoustic neurinoma: MR findings. Radiat Med. 1996, 14:65-9.

7. Murphy ES, Suh JH: Radiotherapy for vestibular schwannomas: a critical review . Int J Radiat Oncol Biol Phys. 2011, 79:985-97. 10.1016/j.ijrobp.2010.10.010

8. Arístegui Ruiz MÁ, González-Orús Álvarez-Morujo RJ, Oviedo CM, Ruiz-Juretschke F, García Leal R, Scola Yurrita B: [Surgical treatment of vestibular schwannoma. Review of 420 cases] . Acta Otorrinolaringol Esp. 2016, 67:201-11. 10.1016/j.otorri.2015.09.003

9. Lanman TH, Brackmann DE, Hitselberger WE, Subin B: Report of 190 consecutive cases of large acoustic tumors (vestibular schwannoma) removed via the translabyrinthine approach. J Neurosurg. 1999, 90:617-23. 10.3171/jns.1999.90.4.0617

10. Barker FG 2nd, Carter BS, Ojemann RG, Jyung RW, Poe DS, McKenna MJ: Surgical excision of acoustic neuroma: patient outcome and provider caseload. Laryngoscope. 2003, 113:1332-43. 10.1097/00005537200308000-00013

11. Chou D, Sampath P, Brem H: Hemorrhagic vestibular schwannoma: an unusual clinical entity. Case report . Neurosurg Focus. 1998, 5:9. 10.3171/foc.1998.5.5.10

12. Yonemitsu T, Niizuma H, Kodama N, Fujiwara S, Suzuki J: Acoustic neurinoma presenting as subarachnoid hemorrhage. Surg Neurol. 1983, 20:125-30. 10.1016/0090-3019(83)90462-7

13. Mathkour M, Helbig B, McCormack E, Amenta PS: Acute presentation of vestibular schwannoma secondary to intratumoral hemorrhage: a case report and literature review. World Neurosurg. 2019, 129:157-63. 10.1016/j.wneu.2019.05.075

14. Piccirillo E, Wiet MR, Flanagan S, Dispenza F, Giannuzzi A, Mancini F, Sanna M: Cystic vestibular schwannoma: classification, management, and facial nerve outcomes. Otol Neurotol. 2009, 30:826-34. 10.1097/MAO.0b013e3181b04e18

15. Chee CP, Bailey IC, Refsum SE: Spontaneous massive haemorrhage into acoustic neuroma during anticoagulation therapy. Br J Neurosurg. 1987, 1:489-93. 10.3109/02688698708999641

16. Carlson ML, Tombers NM, Driscoll CLW, et al.: Clinically significant intratumoral hemorrhage in patients with vestibular schwannoma. Laryngoscope. 2017, 127:1420-26. 10.1002/lary.26193

17. McCoyd K, Barron KD, Cassidy RJ: Acoustic neurinoma presenting as subarachnoid hemorrhage: case report. J Neurosurg. 1974, 41:391-93. 10.3171/jns.1974.41.3.0391

18. Shephard RH, Cheeks RE: Subarachnoid haemorrhage and acoustic neuroma. J Neurol Neurosurg Psychiatry. 1981, 44:1057. 10.1136/jnnp.44.11.1057

19. Goetting MG, Swanson SE: Massive hemorrhage into intracranial neurinomas. Surg Neurol. 1987, 27:168-72. 10.1016/0090-3019(87)90290-4

20. Arienta C, Caroli M, Crotti FM: Subarachnoid haemorrhage due to acoustic neurinoma - case report and review of the literature. Neurochirurgia (Stuttg). 1988, 31:162-5. 10.1055/s-2008-1053927 